\title{
Exact BER Analysis for M-QAM Modulation with Transmit Beamforming under Channel Prediction Errors
}

\author{
Eduardo Martos-Naya, José F. Paris, Unai Fernández-Plazaola, and Andrea J. Goldsmith, Fellow, IEEE
}

\begin{abstract}
Significant throughput improvements can be obtained in multiple-input multiple-output (MIMO) fading channels by merging beamforming at the transmitter and maximal ratio combining (MRC) at the receiver. In general, accurate channel state information (CSI) is required to achieve these performance gains. In this paper, we analyze the impact of channel prediction error on the bit error rate (BER) of combined beamforming and MRC in slow Rayleigh fading channels. Exact closed-form BER expressions are obtained in terms of elementary functions. Numerical results show that imperfect CSI causes little BER degradation using channel prediction of moderate complexity.
\end{abstract}

Index Terms-Multiple input multiple output (MIMO) systems, beamforming, maximal ratio combining (MRC), imperfect channel state information (ICSI), channel prediction, bit error rate (BER).

\section{INTRODUCTION}

M ULTIPLE-INPUT multiple-output (MIMO) systems can considerably increase data rates through spatial multiplexing and significantly improve robustness and coverage through beamforming and diversity combining [1]. The capacity and performance of MIMO systems with multiplexing, beamforming and diversity depends on the availability and accuracy of the channel state information (CSI) at both the transmitter and receiver. The impact of imperfect CSI has been the subject of much recent investigation (see e.g. the special issues [2] and [3]).

This paper focuses on the impact of imperfect CSI on MIMO beamforming, which has been recently addressed in [4], [5], [6], [7]. In particular, [7] determined the pdf, cdf, and moment-generating function (MGF) of the output SNR in transmit beamforming under imperfect CSI. The system model assumed in [7] considers the same CSI to perform both beamforming at the transmitter and MRC at the receiver. However, in this paper we focus on a different system model which considers a more accurate CSI for MRC than for beamforming, as was also considered in [4], [5], [6]. These papers investigated the impact of imperfect CSI on transmit beamforming combined with MRC at the receiver and adaptive modulation. These works do not attempt to obtain exact closed-form BER expressions but rather use approximations, typically based on exponential bounds (e.g. [8, eq. 17]).

Manuscript received February 15, 2007; revised May 25, 2007, September 10, 2007, January 16, 2008, and May 30, 2008; accepted July 31, 2008. The associate editor coordinating the review of this paper and approving it for publication was I. Collings.

E. Martos-Naya, J. F. Paris, and U. Fernández-Plazola are with the Departamento de Ingeniera de Comunicaciones, Universidad de Málaga (email: eduardo@ic.uma.es).

A. J. Goldsmith is with the Department of Electrical Engineering, Stanford University.

Digital Object Identifier 10.1109/T-WC.2008.070192
In contrast, our analysis focuses on obtaining exact BER expressions.

The BER for transmit beamforming will depend on the effective channel gain. If the CSI available to perform beamforming at the transmitter is different from the CSI to perform MRC at the receiver, the pdf of the effective channel gain can seldom be obtained in closed-form. In fact, the wellknown pdf/MGF approach [9] cannot be extended to combined beamforming and MRC analyzed in this paper, and thus alternative analysis techniques are needed.

Under imperfect CSI caused by channel prediction errors, we obtain exact closed-form BER expressions for transmit beamforming using a different approach. Specifically, for a system with fixed power and M-QAM constellation, we compute the exact BER by first calculating the conditional BER, conditioned on the predicted channel, using Proakis' analysis of complex Gaussian quadratic forms [10, Appendix B][11] and then averaging the conditional BER over the distribution of the predicted channel.

The remainder of this paper is organized as follows. Section II describes the system model. In Section III the BER expressions are derived. Section IV presents numerical results which exploit the analytical expressions derived in previous sections. Finally, conclusions are provided in Section V.

\section{SyStem Model}

The system model for MIMO beamforming with MRC is briefly described in this section. Further details on the adopted system model can be found in [4]. We consider $N_{T}$ transmit antennas and $N_{R}$ receive antennas, and the channel is modeled by an $N_{R} \times N_{T}$ complex matrix $\mathbf{H}$, so that each entry $\mathrm{H}_{i, j}$ is the channel coefficient between the $j$ th transmit and the $i$ th receive antennae. These channel coefficients exhibit frequency-flat slowly time-varying fading. The entries $\mathrm{H}_{i, j}$ are assumed independent identically-distributed (i.i.d) complex circularly symmetric normal random variables (RVs), with zero-mean and unity-variance, i.e. $\mathrm{H}_{i, j} \sim \mathcal{C N}(0,1)$. Noise is modeled by an additive $N_{R}$-dimensional vector $\mathbf{n}$, whose entries $n_{k}$ are i.i.d. complex circularly symmetric normal RVs $\sim \mathcal{C N}\left(0, N_{0}\right)$. The received signal can be expressed as

$$
\mathbf{y}=\mathbf{H w}+\mathbf{n},
$$

where $\mathbf{y}$ is the received $N_{R}$ dimensional complex vector and w is the transmitted $N_{T}$ dimensional complex vector.

In our receiver model, as in [4], [5], [6], we assume an imperfect channel prediction to obtain the predicted beamsteering vector which must be fed back to the transmitter. The 
predicted channel $\hat{\mathbf{H}}$ can be expressed as follows

$$
\begin{aligned}
& \hat{\mathbf{H}}=\mathbf{H}-\hat{\boldsymbol{\Xi}}, \quad \text { with } \\
& \left\{\begin{array}{ll}
\hat{\mathrm{H}}_{i, j} & \text { i.i.d. RVs } \sim \mathcal{C N}(0,1-\chi) \\
\hat{\Xi}_{i, j} & \text { i.i.d. RVs } \sim \mathcal{C N}(0, \chi)
\end{array},\right.
\end{aligned}
$$

where $\hat{\boldsymbol{\Xi}}$ is the prediction error matrix and $\chi$ the mean square error. We assume the entries of the predicted channel matrix $\hat{\mathrm{H}}_{i, j}$ and the entries of the prediction error matrix $\hat{\Xi}_{i, j}$ are orthogonal. As in [4], our analysis adopts a slowly timevarying channel model in which the channel response remains invariant along the frame interval.

Using the predicted channel $\hat{\mathbf{H}}$, the optimal beam-steering vector $\hat{\mathbf{v}}$ is the $N_{T}$-dimensional eigenvector corresponding to the largest eigenvalue $\hat{\lambda}$ of matrix $\hat{\mathbf{H}}^{\mathcal{H}} \hat{\mathbf{H}}$ [12], which is given by $\hat{\lambda}=\hat{\mathbf{v}}^{\mathcal{H}} \hat{\mathbf{H}}^{\mathcal{H}} \hat{\mathbf{H}} \hat{\mathbf{v}}$. On each frame, the receiver feeds vector $\hat{\mathbf{v}}$ back to the transmitter to perform beamforming, so that the transmitted vector becomes $\mathbf{w}=\hat{\mathbf{v}} z$, where $z$ is the complex transmitted symbol. The effective channel is an $N_{R}$-dimensional vector defined as $\mathbf{h} \triangleq \mathbf{H} \hat{\mathbf{v}}$ and the predicted effective channel is the vector $\hat{\mathbf{h}} \triangleq \hat{\mathbf{H}} \hat{\mathbf{v}}$, whose square Euclidean norm is $\|\hat{\mathbf{h}}\|^{2}=\hat{\lambda}$. The effective channel can also be expressed as $\mathbf{h} \triangleq \mathbf{H} \hat{\mathbf{v}}=(\hat{\mathbf{H}}+\hat{\mathbf{\Xi}}) \hat{\mathbf{v}}=\hat{\mathbf{h}}+\boldsymbol{\Psi}$, where $\boldsymbol{\Psi}$ is a complex normal $N_{R}$-dimension vector whose entries $\Psi_{k}$, assuming that $\hat{\mathbf{v}}$ is a unitary vector, are i.i.d. RVs $\sim \mathcal{C N}(0, \chi)$ [13, p. 26].

At the receiver, the effective channel vector $\mathbf{h}=\mathbf{H} \hat{\mathbf{v}}$ is assumed perfectly estimated to perform MRC. This assumption is reasonable since the received signals can be stored so that non-causal channel estimation (smoothing) with high accuracy can be performed [14]. The symbol $r$ which results from applying MRC to received vector $\mathbf{y}$ is given by

$$
r \triangleq \frac{\mathbf{h}^{\mathcal{H}} \mathbf{y}}{\|\mathbf{h}\|^{2}}=z+n^{\prime}, \text { with } n^{\prime} \triangleq \frac{(\hat{\mathbf{h}}+\mathbf{\Psi})^{\mathcal{H}} \mathbf{n}}{\|\hat{\mathbf{h}}+\mathbf{\Psi}\|^{2}},
$$

where $n^{\prime}$ is the resultant noise after MRC. As the entries of noise vector $\mathbf{n}$ are i.i.d circularly symmetric Gaussian variables and $\mathbf{h}$ and $\mathbf{n}$ are mutually independent, it is straightforward to show that $n^{\prime}$ is circularly symmetric too.

\section{BER ANALYSIS}

One approach to calculate the BER from (3) could be to average the conditional BER over the effective SISO channel gain $\|\mathbf{h}\|^{2}$. Unfortunately, the pdf of the effective SISO channel gain is unknown to the best of the authors' knowledge. For this reason we use an alternative analysis method. First, we compute the BER conditioned on the predicted effective channel vector $\hat{\mathbf{h}}$, using the Proakis' analysis of Gaussian quadratic forms [10, Appendix B][11]. As is shown in this section, this conditional BER expression only depends on $\hat{\lambda}=\|\hat{\mathbf{h}}\|^{2}$, whose pdf is directly related to the well-known pdf of $\lambda$.

The BER analysis presented in this section considers L-PAM or square M-QAM $\left(M=L^{2}\right)$ with independent bitmapping for in-phase and quadrature components, e.g, Gray mapping. Under these assumptions and reminding that the resultant noise $n^{\prime}$ is circularly symmetric, the BER can be expressed, in a similar way to [15][16], as

$$
B E R=\sum_{n=1}^{L-1} \omega(n) \mathcal{I}(n),
$$

where $\mathcal{I}(n)$ are called components of error probability (CEP) and the $\omega(n)$ are coefficients dependent on the constellation mapping. The CEPs are defined as $\mathcal{I}(n)=\operatorname{Pr}\left\{\Re\left\{n^{\prime}\right\}>(2 n-1) d\right\}$, where $d$ is the minimum distance between the symbol and the decision boundary and can be expressed as a function of the constellation energy $E_{s}$ as $d=\sqrt{\frac{6 E_{s}}{M-1}}$ for M-QAM constellation and $d=\sqrt{\frac{12 E_{s}}{L^{2}-1}}$ for L-PAM constellation. The coefficients $\omega(n)$ can be obtained using the method described in [16] or directly computed for Gray mapping as in [15].

In Section III-A we derive the CEP conditioned on the predicted effective channel vector $\hat{\mathbf{h}}$. In Section III-B, using the previous results, we obtain both the conditional BER and the BER averaged over $\hat{\lambda}$.

\section{A. Conditional CEP}

In this section we derive, for our system model, the CEP conditioned on a predicted channel state (CCEP), that can be expressed as

$$
\mathcal{I}(n ; \hat{\mathbf{h}})=\operatorname{Pr}\left\{\Re\left\{n^{\prime}\right\}>(2 n-1) d \mid \hat{\mathbf{h}}\right\} .
$$

In order to obtain the CCEP we use Proakis' analysis of complex Gaussian quadratic forms [10, Appendix B], more specifically, we adopt the compact expressions presented in [11] and restated in Table I.

According to our system model, when the symbol $z=s$ is transmitted, each entry of the received signal $y_{k}$ and each entry of the effective channel $h_{k}$ are

$$
\begin{gathered}
y_{k}=h_{k} s+n_{k}=\left(\hat{h}_{k}+\Psi_{k}\right) s+n_{k}, \\
h_{k}=\hat{h}_{k}+\Psi_{k},
\end{gathered}
$$

where both $y_{k}$ and $h_{k}$ are complex normal variables conditioned on the predicted effective $\hat{h}_{k}$. Note that $\hat{h}_{k}$ is assumed orthogonal to the channel error $\Psi_{k}$ (e.g. $\hat{h}_{k}$ is predicted using a FIR Wiener filter) and therefore, the probability distribution of $\Psi_{k}$ is independent of the $\hat{h}_{k}$ value. Defining the sum of quadratic forms $D=\sum_{k=1}^{N_{R}} \mathbf{x}_{k}^{\mathcal{H}} \mathbf{Q} \mathbf{x}_{k}$, where

$$
\mathbf{x}_{k}=\left[\begin{array}{l}
y_{k} \\
h_{k}
\end{array}\right], \quad \mathbf{Q}=\left[\begin{array}{cc}
0 & -1 / 2 \\
-1 / 2 & \Re\{s\}+(2 n-1) d
\end{array}\right],
$$

we have

$$
\begin{aligned}
& \operatorname{Pr}\{D<0\}= \\
& \operatorname{Pr}\left\{-\Re\left\{\mathbf{h}^{\mathcal{H}} \mathbf{y}\right\}+(\Re\{s\}+(2 n-1) d)\|\mathbf{h}\|^{2}<0 \mid \hat{\mathbf{h}}\right\}=(9) \\
& \operatorname{Pr}\left\{\Re\left\{n^{\prime}\right\}>(2 n-1) d \mid \hat{\mathbf{h}}\right\}=\mathcal{I}(n ; \hat{\mathbf{h}}) .
\end{aligned}
$$

In Table I we present the expressions to obtain this probability. Using the quadratic form matrix $\mathbf{Q}$, the mean vector $\mathbf{m}_{k}$ and the covariance matrix $\mathbf{R}$ of vector $\mathbf{x}_{k}$, we can calculate the parameters $a, b, \eta$ and $C_{l}$ necessary to obtain this probability. From (6) and (7), we obtain the mean and the covariance 
TABLE I

PROBABILITY COMPUTATION OF THE GAUSSIAN QUADRATIC FORM.

\begin{tabular}{|c|c|}
\hline$\overline{\text { FUNCTIONS AND PARAMETERS }}$ & DEFINITIONS \\
\hline$D$ & $\sum_{k=1}^{L} \mathbf{x}_{k}^{\mathcal{H}} \mathbf{Q} \mathbf{x}_{k}$ \\
\hline $\mathbf{m}_{k}$ & $\mathrm{E}\left\{\mathbf{x}_{k}\right\}$ \\
\hline $\mathbf{R}$ & $\mathrm{E}\left\{\left(\mathbf{x}_{k}-\mathbf{m}_{k}\right)\left(\mathbf{x}_{k}^{\mathcal{H}}-\mathbf{m}_{k}^{\mathcal{H}}\right)\right\}$ \\
\hline$\left\{\delta_{i}\right\}_{i=1,2}$ & $\frac{1}{2} \operatorname{tr}(\mathbf{R Q}) \pm \sqrt{\left(\frac{1}{2} \operatorname{tr}(\mathbf{R Q})\right)^{2}-\operatorname{det}(\mathbf{R Q})}$ \\
\hline$\eta$ & $\left|\frac{\delta_{1}}{\delta_{2}}\right|$ \\
\hline \multirow{2}{*}{$a$} & $\sqrt{2 \delta_{2}\left(\sum_{k=1}^{L} \mathbf{m}_{k}^{\mathcal{H}}\left[\mathbf{Q}-\delta_{1} \mathbf{R}^{-1}\right] \mathbf{m}_{k}\right)}$ \\
\hline & $\sqrt{\left(\delta_{1}-\delta_{2}\right)^{2}}$ \\
\hline \multirow{2}{*}{$b$} & $\longdiv { 2 \delta _ { 1 } ( \Sigma _ { k = 1 } ^ { L } \mathbf { m } _ { k } ^ { \mathcal { H } } [ \mathbf { Q } - \delta _ { 2 } \mathbf { R } ^ { - 1 } ] \mathbf { m } _ { k } ) }$ \\
\hline & $\sqrt{\left(\delta_{1}-\delta_{2}\right)^{2}}$ \\
\hline$C_{l}(a, b, \eta)$ & $\begin{array}{ll}-1+\frac{1}{(1+\eta)^{2 L-1}} \sum_{k=0}^{L-1}\left(\begin{array}{c}2 L-1 \\
k\end{array}\right) \eta^{k}, & l=0 \\
\frac{1}{(1+\eta)^{2 L-1}} \sum_{k=0}^{L-1-l}\left(\begin{array}{c}2 L-1 \\
k\end{array}\right)\left[\left(\frac{b}{a}\right)^{l} \eta^{k}-\left(\frac{a}{b}\right)^{l} \eta^{2 L-1-k}\right], & l \neq 0\end{array}$ \\
\hline PROBABILITY & EXPRESSION \\
\hline $\operatorname{Pr}\{D<0\}$ & $\mathrm{Q}_{1}(a, b)+\sum_{l=0}^{L-1} C_{l}(a, b, \eta) \mathrm{I}_{l}(a b) \exp \left\{-\frac{1}{2}\left(a^{2}+b^{2}\right)\right\}$ \\
\hline
\end{tabular}

matrix of the vector $\mathbf{x}_{k}$ as

$$
\mathbf{m}_{k}=\left[\begin{array}{c}
\hat{h}_{k} s \\
\hat{h}_{k}
\end{array}\right], \quad \mathbf{R}=\left[\begin{array}{cc}
\chi|s|^{2}+N_{0} & \chi s \\
\chi s^{*} & \chi
\end{array}\right] .
$$

Using Table I expressions, equations (8) and (10), and taking into account $\sum_{k=1}^{N_{R}}\left|\hat{h}_{k}\right|^{2}=\hat{\lambda}$, the constants $a, b$ and $\eta$ are given by

$$
\begin{aligned}
& a=a_{n} \sqrt{\hat{\lambda}}, \text { with } \\
& a_{n}=\sqrt{\frac{1+2 \kappa_{n} \bar{\gamma} \chi-2 \sqrt{\kappa_{n} \bar{\gamma} \chi\left(\kappa_{n} \bar{\gamma} \chi+1\right)}}{2\left(\kappa_{n} \bar{\gamma} \chi+1\right) \chi}}, \\
& b=b_{n} \sqrt{\hat{\lambda}}, \text { with } \\
& b_{n}=\sqrt{\frac{1+2 \kappa_{n} \bar{\gamma} \chi+2 \sqrt{\kappa_{n} \bar{\gamma} \chi\left(\kappa_{n} \bar{\gamma} \chi+1\right)}}{2\left(\kappa_{n} \bar{\gamma} \chi+1\right) \chi}} \\
& \eta=\eta_{n}=1+2 \kappa_{n} \bar{\gamma} \chi+2 \sqrt{\kappa_{n} \bar{\gamma} \chi\left(\kappa_{n} \bar{\gamma} \chi+1\right)}
\end{aligned}
$$

where the average SNR is defined as $\bar{\gamma} \triangleq \frac{E_{S}}{N_{0}}$, and the constant $\kappa_{n} \triangleq \frac{3(2 n-1)^{2}}{2(M-1)}$ for M-QAM and $\kappa_{n} \triangleq \frac{3(2 n-1)^{2}}{\left(L^{2}-1\right)}$ for L-PAM. Note that, after some algebra manipulations, it can be shown that $\eta_{n}=b_{n} / a_{n}$.

Substituting the parameters $a, b$, and $C_{l}$ in the expression of $\mathrm{P}\{D<0\}$ that appears in Table I, the CCEP is expressed as

$$
\begin{aligned}
& \mathcal{I}(n ; \hat{\mathbf{h}})=\mathcal{I}(n ; \hat{\lambda})=\mathrm{Q}_{1}\left(a_{n} \sqrt{\hat{\lambda}}, b_{n} \sqrt{\hat{\lambda}}\right)+ \\
& \sum_{l=0}^{N_{R}-1} C_{l}\left(a_{n}, b_{n}\right) \mathrm{I}_{l}\left(a_{n} b_{n} \hat{\lambda}\right) \exp \left[-\frac{\hat{\lambda}}{2}\left(a_{n}^{2}+b_{n}^{2}\right)\right],
\end{aligned}
$$

where $\mathrm{Q}_{1}$ is the Marcum-Q function, and $\mathrm{I}_{l}$ is the $l$-th order modified Bessel function of the first kind.

In the absence of channel prediction error, i.e. $\Psi_{k}=0$, the resultant noise after MRC $n^{\prime}$, whose expression is given in (3), is a Gaussian variable conditioned on $\hat{\mathbf{h}}$ whose variance is $N_{0} / \hat{\lambda}$. Thus, the calculation of CCEP is equivalent to the BER calculation for a standard Gaussian noise channel, resulting in

$$
\mathcal{I}(n ; \hat{\lambda})=\mathrm{Q}\left(\sqrt{2 \hat{\lambda} \kappa_{n} \bar{\gamma}}\right),
$$

where Q is the Gaussian Q-function.

\section{B. Conditional and average BER expressions}

The CCEP calculated in the previous section allows us to obtain the conditional BER (CBER). This probability represents the BER conditioned on the predicted effective channel gain $\hat{\lambda}$, i.e, the BER under imperfect channel state information (CSI). Introducing (12) in (4) yields

$$
C B E R(\hat{\lambda})=\sum_{n=1}^{L-1} \omega(n) \mathcal{I}(n ; \hat{\lambda}) .
$$




$$
\begin{aligned}
& \text { BER }=\sum_{n=1}^{L-1} \sum_{k=1}^{N_{1}} \sum_{r=N_{2}-N_{1}}^{\left(N_{2}+N_{1}-2 k\right) k} \frac{\omega(n) B_{k, r}}{k^{r+1}} \times \\
& \left\{\frac { r ! } { k ^ { r + 1 } } \left\{1+\frac{b_{n}^{2}}{s_{n, k}} \sum_{m=0}^{r}(m+1)\left(\frac{2 k}{s_{n, k}}\right)^{m}\left[\frac{a_{n}^{2}}{s_{n, k}}{ }_{2} F_{1}\left(\frac{m+2}{2}, \frac{m+2}{2}+\frac{1}{2} ; 2 ; \frac{4 a_{n}^{2} b_{n}^{2}}{s_{n, k}^{2}}\right)-\right.\right.\right. \\
& \left.\left.\frac{1}{1+m}{ }_{2} F_{1}\left(\frac{m+1}{2}, \frac{m+1}{2}+\frac{1}{2} ; 1 ; \frac{4 a_{n}^{2} b_{n}^{2}}{s_{n, k}^{2}}\right)\right]\right\}+\sum_{l=0}^{N_{R}-1} \frac{(r+l) !}{\left(a_{n} b_{n}\right)^{r+1}} \frac{1}{l !}\left(\frac{w_{n, k}-1}{w_{n, k}+1}\right)^{l / 2}\left(\frac{w_{n, k}+1}{2}\right)^{r} \times \\
& \left.{ }_{2} F_{1}\left(-r,-r+l ; l+1 ; \frac{w_{n, k}-1}{w_{n, k}+1}\right) \sqrt{\left(w_{n, k}^{2}-1\right)^{r+1}}\right\},
\end{aligned}
$$

The BER is obtained by averaging the CBER over the predicted eigenvalue $\hat{\lambda}$ as

$$
B E R=\sum_{n=1}^{L-1} \omega(n) \int_{0}^{\infty} \mathcal{I}(n ; \hat{\lambda}) p(\hat{\lambda}) d \hat{\lambda}
$$

Using the fact that the pdf of the largest eigenvalue of complex Wishart matrices can be expressed as a weighted sum of elementary Gamma pdfs [17], the pdf of $\hat{\lambda}$ is given by

$$
p(\hat{\lambda})=\sum_{k=1}^{N_{1}} \sum_{r=N_{2}-N_{1}}^{\left(N_{2}+N_{1}-2 k\right) k} B_{k, r} \frac{\hat{\lambda}^{r}}{(1-\chi)^{r+1}} \exp \left(\frac{-k \hat{\lambda}}{1-\chi}\right),
$$

where $N_{1} \triangleq \min \left\{N_{T}, N_{R}\right\}, N_{2} \triangleq \max \left\{N_{T}, N_{R}\right\}$ and the coefficients $B_{k, r}$ are easily deducted from the expression [17, eq.10].

Substituting equations (12) and (16) in (15), using the expression [18, eq.8.772-3] and the integrals given in [18, eq.8.914-1] and [19], we can obtain, after some algebraic manipulation, the closed-form BER expression (17) at the top of the page, where ${ }_{2} F_{1}$ is the Gauss hypergeometric function, $s_{n, k}=a_{n}^{2}+b_{n}^{2}+2 k$ and $w_{n, k}=\left(1-\frac{4 a_{n}^{2} b_{n}^{2}}{s_{n, k}^{2}}\right)^{-1 / 2}$. Note that function ${ }_{2} F_{1}$ in (17) can be expressed as a finite sum of elementary functions for the values of its arguments, although it is not shown here for compactness reasons.

For the perfect CSI case, substituting expression (13) and (16) in (15) the BER under perfect CSI is obtained as

$$
\begin{aligned}
& B E R=\sum_{n=1}^{L-1} \sum_{k=1}^{N_{1}} \sum_{r=N_{2}-N_{1}}^{\left(N_{2}+N_{1}-2 k\right) k} \frac{\omega(n) B_{k, r} r !}{2 k^{r+1}} \times \\
& \left.1-\sqrt{\frac{\kappa_{n} \bar{\gamma}}{k+\kappa_{n} \bar{\gamma}}} \sum_{l=0}^{r}\left(\begin{array}{c}
2 l \\
l
\end{array}\right)\left(\frac{k}{4\left(k+\kappa_{n} \bar{\gamma}\right)}\right)^{l}\right],
\end{aligned}
$$

where we use the fact that the required integral over $\hat{\lambda}$ is formally identical to [9, Eq. 5.18].

\section{Numerical Results}

The numerical results are obtained from analytical expressions and simulations assuming a prediction subsystem similar to that described in [4]. We consider Jakes' model for the channel time-correlation and Wienner filtering to predict the CSI. The numerical values for the system parameters are: carrier frequency $f_{c}=3 \mathrm{GHz}$, mobile speed $v=36 \mathrm{~km} / \mathrm{h}$, feedback delay $\tau=1.28 \mathrm{~ms}$ and frame interval $T=0.64 \mathrm{~ms}$, which

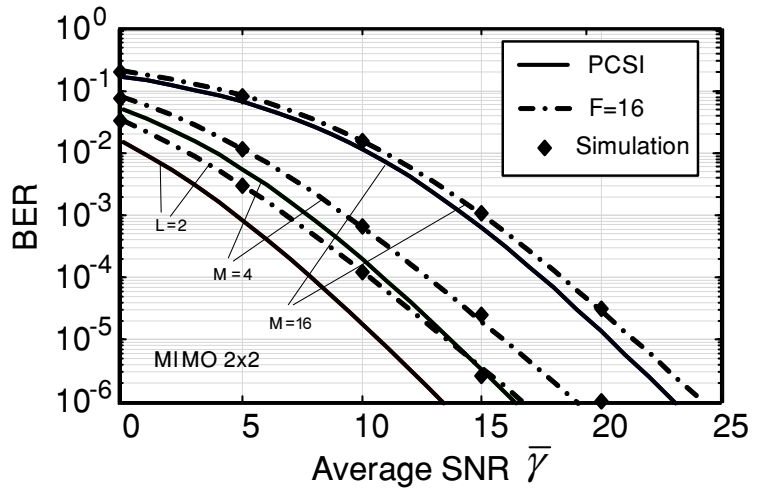

Fig. 1. BER as a function of average SNR $\bar{\gamma}$ for different constellations under perfect CSI (PCSI) and imperfect prediction ( $F=16$ taps).

e.g. could correspond to a system with a symbol frequency $f_{S}=100 \mathrm{kHz}$ and 64 symbols per frame.

Figure 1 shows the BER for a $2 \times 2$ MIMO system as a function of the average SNR $\bar{\gamma}$ for some L-PAM and M-QAM constellations in two cases: perfect CSI using equation (18) and imperfect CSI using (17) with a 16-tap FIR Wiener prediction filter. In the last case, we assume channel estimation based on pilot symbol assisted modulation (PSAM) and the mean square error $\chi$ is computed as described in [4, section II-B]. As a double check, simulation results for the imperfect CSI case are also superimposed in the figure. There is an unavoidable channel prediction error floor independent of the number of filter taps and, thus, the BER for perfect CSI cannot be achieved. Note that at around $\mathrm{BER} \approx 10^{-3}$ the relative SNR losses due to the channel prediction error for the considered system parameters are about $2.5 \mathrm{~dB}$ for BPSK, $1.5 \mathrm{~dB}$ for 4-QAM and less than $1 \mathrm{~dB}$ for 16-QAM.

In Figure 2 the influence of antenna configuration on the BER is depicted for 16-QAM. Obviously in the SISO case the channel prediction error does not affect the BER performance. Moreover, the 2x4 and 4x2 MIMO systems show the same BER for perfect CSI. This is because, if perfect CSI is assumed, the BER performance for different antenna configurations only depends on the pdf of $\hat{\lambda}$, which is the same for the $2 \times 4$ and $4 \times 2$ MIMO systems. However, for the imperfect CSI case the BER performance is better for $2 \times 4$ MIMO systems. 


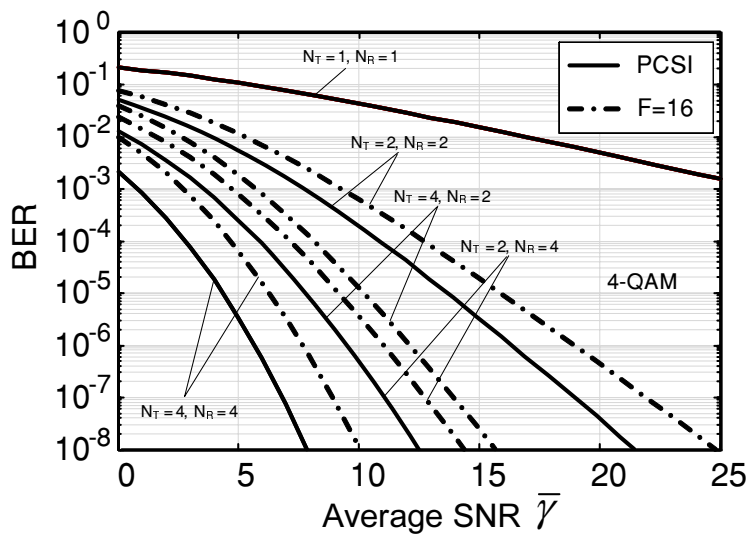

Fig. 2. BER as a function of average SNR $\bar{\gamma}$ for different antenna configurations under perfect CSI (PCSI) and imperfect prediction ( $F=16$ taps).

\section{CONCLUSIONS}

Exact closed-form BER expressions for MIMO beamforming with MRC systems under channel prediction errors have been derived. These results allow us to analyze the performance of $L$-PAM and square $M$-QAM using practical estimation methods under Rayleigh fading. The system performance has been analyzed for different scenarios with different values of the mobile speed, number of prediction filter taps, adaptation delay, number of transmitter and receiver antennas, constellation size and average SNR. Our results indicate that practical constraints imposed by channel prediction do not significantly degrade BER performance.

\section{ACKNOWLEDGMENT}

This work is partially supported by the Spanish Government under project TEC2007-67289/TCM and by AT4 wireless. The work of A. Goldsmith was supported in part by LG Electronics.

\section{REFERENCES}

[1] A. Goldsmith, Wireless Communications. Cambridge University Press, 2005.

[2] M. Shafi, D. Gesbert, D. S. Shiu, P. J. Smith, and W. H. Tranter, "MIMO systems and applications II," IEEE J. Select. Areas Commun., vol. 21, no. 5, June 2003.

[3] D. Palomar, T. Davidson, S. Barbarossa, A. Goldsmith, and G. Giannakis, "Optimization of MIMO transceivers for realistic communication networks: Challenges and opportunities," IEEE J. Select. Areas Commun., vol. 25, no. 7, Sept. 2007.

[4] S. Zhou and G. Giannakis, "How accurate channel prediction needs to be for transmit-beamforming with adaptive modulation over Rayleigh MIMO channels?" IEEE Trans. Wireless Commun., vol. 3, no. 4, pp. 1285-1294, July 2004.

[5] A. Maaref and S. Aissa, "Generalized performance analysis of adaptive PSAM-based transmit-beamforming for wireless MIMO systems," in Proc. Vehicular Technology Conf. (VTC), vol. 5, Melbourne, Vic., May 2006, pp. 2578-2582.

[6] J. F. Paris and A. J. Goldsmith, "Adaptive modulation for MIMO beamforming under average BER constraints and imperfect CSI," in Proc. IEEE Int. Conf. Communications (ICC), vol. 3, Istanbul, June 2006, pp. 1312-1317.

[7] Y. Chen and C. Tellambura, "Performance analysis of maximum ratio transmission with imperfect channel estimation," IEEE Commun. Lett., vol. 9, no. 4, pp. 322-324, Apr. 2005.

[8] A. J. Goldsmith and S.-G. Chua, "Variable-rate variable-power MQAM for fading channels," IEEE Trans. Commun., vol. 45, no. 10, pp. 12181230, Oct. 1997.

[9] M. K. Simon and M.-S. Alouini, Digital Communication over Fading Channels, 2nd ed. New York: Wiley, 2005.

[10] J. Proakis, Digital Communications, 4th ed. McGraw-Hill, 2001

[11] U. Fernández-Plazaola, E. Martos-Naya, and J. Paris, "Another look at the analysis of the characteristic function of complex Gaussian quadratic forms," Math. Problems in Eng., submitted for publication.

[12] S. Zhou and G. B. Giannakis, "Optimal transmitter eigen-beamforming and space-time block coding based on channel correlations," IEEE Trans. Inform. Theory, vol. 49, no. 7, pp. 1673-1690, July 2003.

[13] A. Tulino and S. Verdú, Random Matrix Theory and Wireless Cоттиnications. Hanover: Now Publishers Inc., 2004.

[14] G. E. Oien, H. Holm, and K. J. Hole, "Impact of channel prediction on adaptive coded modulation performance in rayleigh fading," IEEE Trans. Veh. Technol., vol. 53, no. 3, pp. 758-769, May 2004.

[15] K. Cho and D. Yoon, "On the general BER expression of one- and twodimensional amplitude modulations," IEEE Trans. Commun., vol. 50, no. 7, pp. 1074-1080, July 2002.

[16] L. Cao and N. C. Beaulieu, "Closed-form BER results for MRC diversity with channel estimation errors in Ricean fading channels," IEEE Trans. Wireless Commun., vol. 4, no. 4, pp. 1440-1447, July 2005.

[17] A. Maaref and S. Aissa, "Closed-form expressions for the outage and ergodic Shannon capacity of MIMO MRC systems," IEEE Trans. Commun., vol. 53, no. 7, pp. 1092-1095, July 2005.

[18] I. S. Gradshteyn and I. M. Ryzhik, Table of Integrals, 5th ed. San Diego: Academic Press, 2000.

[19] M. K. Simon and M. S. Alouini, "Some new results for integrals involving the generalized Marcum Q function and their application to performance evaluation over fading channels," IEEE Trans. Wireless Commun., vol. 2, no. 4, pp. 611-615, July 2003. 